\title{
L'Algérie et les défis du commerce mondial
}

\author{
Khayreddine BELAAZE, \\ Mohamed KhiderUniversity, ALGERIA \\ Rabah KHOUNI \\ Mohamed KhiderUniversity, ALGERIA
}

\section{Introduction:}

L'Algérie a activement cherché à s'engager dans le système commercial multilatéral à travers des négociations bilatérales et multilatérales au sein de l'Organisation mondiale du commerce, Mais le manque de réceptivité aux règles de l'OMC a empêché d'atteindre cet objectif, Cela a été décidé de s'engager dans des accords commerciaux régionaux pour tirer les gains de création et détournement de commerce.

De ce point de vue, l'Algérie a signé un accord d'association avec l'union européen, et un accord pour établir une zone de libre-échanges avec les pays arabes - la grande zone arabe de liber échanges-, pour le but de réadaptation les entreprises algériennes et faire accroît leur compétitivité.

Mais le système commercial multilatéral est on crise, en raison du fait que tous les accords commerciaux régionaux sont incompatibles avec la clause de la nation la plus favorisée, certains doutes restent émis que les tarifs accordés aux partenaires dans le cadre de l'accord régional sont Inférieurs à ceux accordés dans le cadre d'OMC.

L'objectif de cette étude est de tenter de comprendre la place d'Algérie dans ce défi, dans les prochains années l'Algérie rejoindra l'OMC, c'est ce qui va être mis à s'interroger sur l'avenir des accords régionaux, Parce que nous allons expliquer et par preuve comment les accords commerciaux régionaux ne concordent pas et les règles d'OMC.

Cet article s'organise en trois parties, dans le premier on donne une analyse sur l'évolution de système commercial multilatéral, de charte de la Havane à l'OMC, dans un second temps nous offrons une explication sur les accords commerciaux régionaux et sont conflit avec les règles de l'OMC.

Nous essaierons d'exposer par l'analyse le cas de l'Algérie, a partir de la structure 
de commerce extérieur et puis nous nous focaliserons sur les étapes d'avancement d'accession de l'Algérie à l'OMC, et ensuit sur les accords régionaux de liber échanges.

\section{1- Le système commercial multilatéral : Du GATT à l'OMC}

L'idée de la libéralisation du commerce international et l'élimination des restrictions douanières n'étaient pas en conséquence de la Charte de La Havane, ou l'Accord général sur les tarifs douaniers et le commerce, mais à la fin de la Première Guerre mondiale , par la déclaration de la président Wilson, ou demander l'abolition des barrières et les mesures commerciales qui faussant les échanges, Et les États-Unis a été l'un des partisans de la libéralisation du commerce international et l'abolition des restrictions au commerce, en particulier après la signature de le «Reciprocal Trade Agreements Act» et une série d'accords bilatéraux «Cordelle Hull Agreements » en $1934^{(1)}$, Mais la première proposition pour la création d'une organisation internationale spécialisée dans l'arbitrage international dans des différends commerciaux, a été le 1 Août 1942 connu sous le nom de projet d'union commerciale par James Meade .

C'est un fait que le projet d'Union commerciale dont James Meade rédige trois versions successives durant l'été 1942 tire les grandes lignes de ce que sera quelques années plus tard la charte de la Havane $[\ldots]^{(2)}$.

Le projet incluait 22 paragraphe vise à réduire les restrictions douanières et régler le déséquilibre des paiements et de stimuler la croissance de la demande sur les marchés mondiaux.

\section{1-1 - Charte de la HAVANE : pour une organisation internationale du commerce}

Après la création des institutions de Bretton Woods (Banque mondiale et Fonds monétaire international), A dû être Mise en place d'une institution internationale complète le triangle Nouvel Ordre Mondial - The New World Order - Intéressée à développer les bases des politiques commerciales des Etats membres, Qui ont déjà fait en 21 novembre 1947 Les délégués de 56 pays se sont réunis a La Havane, Cuba, D'après les recommandations du Conseil économique et social des Nations Unies qui a recommandé D'organiser une conférence internationale sur le commerce et l'emploi, l'acte final de la charte de La Havane contient de 106 articles, Divisée sur neuf chapitres traitent: l'emploi et activité économique, développement économiques, politique commerciale, les accords intergouvernementaux sur les produits de base, l'organisation international du commerce et règlement des différends. 


\section{1-2-Accord général sur les tarifs douaniers et le commerce : le GATT}

Dans la même période de la charte de Havane Il y avait des réunions parallèles, Préparé le terrain pour la création de GATT, après une série de conférences à New York et à Londres, et enfin à Genève, 23 pays a ratifié l'Accord général sur les tarifs douaniers et le commerce qui est entré en vigueur le 30 Juin 1948, cet accord a remplacé la charte de Havane, et a appelé «protocole d'application provisoire », ce protocole qui a duré près d'un demi-siècle.

L'Accord général sur les tarifs douaniers et le commerce inclus bon nombre des principes, Visant à réduire les droits de douane et élimination des restrictions quantitatives dans le but d'encourager les échanges commerciaux et parvenir ainsi un développement économique, généraux de la Convention de l'accord on trouve:

- Traitement général de la nation la plus favorisée,

- Traitement national,

- La libéralisation des échanges et soutien le commerce équitable,

- La transparence et la gouvernance,

- Le développement économique,

Et comme il est indiqué dans la partie 4, sous le titre: Commerce et développement, L'article 18 sélectionné le Soutien de l'État pour le développement économique, L'article 36 a également souligné les objectifs de développement du présent accord qui vise à élever le niveau de vie, le développement progressif des économies des Etats membres, en matière de recettes des exportations pourrait jouer un rôle dans le développement économique, comme il est indiqué au paragraphe $\mathrm{E}$, «le commerce international considéré comme instrument de progrès économique et social» ${ }^{(3)}$.

De 1948 à 1994, l'Accord général sur les tarifs douaniers et le commerce (GATT), Connu huit cycles de négociations, Les premiers cycles de négociations commerciales (Genève 1947, Annecy 1949, Torquay 1951, Genève 1956) étaient axés sur la réduction des droits de douane, le Kennedy Round 1964-1967 a abouti à l'Accord antidumping du GATT et à une section sur le développement, Dans les années 70, le Tokyo Round a été la première tentative majeure visant à remédier aux obstacles au commerce autres que les droits de douane et à améliorer le système $^{(4)}$, la huitième et la dernière cycle qui a duré de 1986 à 1994 a Punta del Este (Uruguay) a débouché sur la création de l'OMC et l'adoption un nouveau ensemble d'accords. 


\section{1-3-L'organisation mondiale du commerce}

Après demi-siècle dans l'accord général sur les tarifs douaniers et le commerce, et en septembre 1986 à Punta Del Este, un programme de négociation a été lancé, Les négociations touché les points suivants : le commerce des services et la propriété intellectuelle, les produits agricoles et les textiles, système de règlement des différends, mais l'agriculture et la création d'une nouvelle institutiondevenir un source de conflit, Les membres devons attendre Le 15 avril 1994 Lorsque l'accord a été signé qui était officiellement dénommé l'accord de Marrakech, en janvier 1995, les accords entrent en vigueur et l'OMC est créée.

Se réunit habituellement une fois tous les deux ans, Les Conférences ministérielles de l'OMC rassemble tous les Membres, l'Organisation mondiale du commerce connaissait huit conférence ministérielle jusqu'à 2012, Un neuvième conférence est prévu à Bali, en Indonésie le 3-6 décembre 2013, Mais le plus important conférence était à Doha-Qatar, Où surnommé le Cycle de Doha pour le développement,

il consiste à renforcer l'intégration des pays en développement dans les échanges mondiaux... l'objectif des négociations portant sur les biens est de réduire, et lorsque cela est approprié, supprimer les tarifs douaniers, ce qui recouvre la réduction ou l'élimination des crêtes tarifaires, des tarifs élevés et de la progressivité des droits, mais également des barrières non-tarifaires, en particulier celles s'appliquant à des produits intéressants à exporter pour les pays en développement. ${ }^{(5)}$

Les principales fonctions de l'OMC sont : les négociations commerciales à partir des Conférences ministérielles qui déroulent une fois tous les deux ans et prendre des décisions sur les domaines suivantes :

- Les marchandises: Accès aux marchés, Agriculture, la politique de l'Antidumping, balance des paiements, droit de douane, sauvegardes et subvention ;

- Accord général sur les tarifs douaniers et le commerce (GATT 1994);

- Accord général sur le commerce des services (GATS);

- Accord sur les aspects des droits de propriété intellectuelle qui touchent au commerce (TRIPS) ;

- accord sur les mesures concernant l'investissement et liées au commerce (TRIMs).

\section{2- $\quad$ Les Accords commerciaux régionaux}

Les accords commerciaux régionaux sont l'un des aspects les plus importants qui caractérisent l'économie mondiale d'jour, Nous pouvons dire avec certitude parce 
que tous les pays du monde sont liés à un ou plusieurs accords commerciaux, qu'elles soient régionales ou préférentiel- Comprendre que tous les accords commerciaux régionaux inclus tous les arrangements accorder des préférences aux pays membres d'une région particulière, comme le Grand zone arabe de libre échange, Mais les arrangements préférentiels dépasser le côté régional tels que le Système de Préférences Généralisé, les zones de libre échange ....

\section{2-1- $\quad$ Les ACR dans le monde}

Selon l'Organisation Mondiale du Commerce en 2010, plus de 300 accords commerciaux régionaux qui sont soit planifiées, ont terminé les négociations, ou qui sont en vigueur.

Comme on l'a dit préalablement, Bien que l'expression «accord commercial régional » soit maintenant largement utilisée, le rapport emploie l'expression plus générique « accord commercial préférentiel » (ACPr), car de nombreux accords ne sont paslimitésaux pays d'une même région. Le rapport traite uniquement des accords préférentiels réciproques- régionaux, bilatéraux ou plurilatéraux. ${ }^{(6)}$

Cependant, la théorie des dominos du régionalisme de Baldwin et l'hypothèse de libéralisation compétitive de Bergsten sont des histoires implicitement dynamiques des l'évolution des accords régionaux, mais la question qui surgit : Comment avoir des accords commerciaux régionaux évolué ?

Pour répondre à la question, il est nécessaire d'examiner les tendances historiques de nouveau système commercial particulièrement après les années 50 , La première vague de régionalisme a eu lieu à la fin des années 1950 et dans les années 1960, Plus précisément dans le continent européen, «Elle a commencé par la création de la Communauté européenne du charbon et de l'acier, en 1951, qui a été suivie, en 1957, par la Communauté économique européenne (CEE) de plus vaste portée, puis s'est étendue aux anciennes colonies à travers un ensemble complexe d'accords commerciaux préférentiels mais non réciproques ... La deuxième vague de régionalisme a commercé à peu près au milieu des années 1980 et s'est prolongée pendant une bonne partie des années 1990... Au cours de la dernière décennie, une nouvelle vague de régionalisme a démarré, là encore sous l'impulsion des grandes puissances commerciales (UE, États-Unis, etc.) mais aussi, pour la première fois, avec la participation de nombreuxpays asiatiques jusque-là ardents défenseurs du multilatéralisme et de la non-discrimination ${ }^{(7)}$.

L'UE un le plus grand bloc régional de monde, $86 \%$ de son volume de commerce extérieur se réalise entre les pays membre de l'UE, Il convient de mentionner ici que la majorité des accords commerciaux régionaux conclus entre les pays du Sud 
et du Nord, Tout d'abord, pour des raisons historiques, la plupart des pays du Sud sont d'anciennes colonies des pays développés, Deuxièmement: en raison de facteurs économiques est l'avantage concurrentiel des produits des pays développés, ainsi que l'abondance des facteurs de production.

Qui a conduit à ces accords de devenir plus en plus des accords préférentiels, JeanMarc Siroen, confirmé cette approche en disant: «Depuis l'accord USA-Israël (1985), les accords entre pays géographiquement éloignés, parfois inter-continentaux, se sont multipliés à l'instar, notamment, de l'Union européenne (pays méditerranéens, Afrique du sud, Chili, etc.),des États-Unis (Maroc, Jordanie, Singapour, etc.), du Chili (Canada, USA, UE, Corée, etc.), trois accords intercontinentaux, aujourd'hui en application, ont été notifiés avant1995. Neuf les ont entre 1996 et 2000 et 27 (sur 79) entre 2001 et 2005. De plus, certains accords signés ne sont pas encore notifiés comme les accords des Etats-Unis avec Bahreïn ou l'Afrique du $\operatorname{Sud}^{(8)}$.

Les ACPr peuvent être classés en fonction : ${ }^{(9)}$

- du niveau de développement (accords entre pays développés ou entre pays en développement seulement, accords entre des pays développés et des pays en développement;

- de la couverture géographique (ACPr intra régionaux ou interrégionaux) à l'intérieur des régions ou entre elles, par exemple l'Asie (Asie de l'Est et de l'Ouest, Océanie), les Amériques (Amérique du Nord, du Sud, centrale et Caraïbes), l'Europe, le Moyen-Orient, l'Afrique et la Communauté d'États indépendants (CEI);

- du type d'ACPr (bilatéraux, plurilatéraux ou entre blocs régionaux);

- du degré d'intégration des marchés (ALE ou union douanière, par exemple) et des domaines visés (marchandises, services, questions réglementaires, par exemple).

L'Algérie a signé plus de 10 arrangements commerciaux préférentiels sous le type de Système généralisé de préférences (SGP) avec l'Union Européen (01 juillet 1971), le japon (01 Aout 1971), le Norvège (01 Octobre 1971), la NouvelleZélande (01 janvier 1972), suisse (01 mars 1972), Australie (01 janvier 1974), Canada (01 juillet 1974), les Etat Unis (01 janvier 1976), Turquie (01 janvier 2002), la fédération de Russie (01 janvier 2010), et plus de 42 accords bilatéraux entre en vigueur le 19 avril 1989, dans schéma de système global de préférences commerciales entre les pays en développement (SGPC). 


\section{2-2- L'OMC et le Régionalisme}

La part de commerce intra-ACPr dans les exportations mondiales de marchandises est plus de $28 \%$ en 1990 , et plus de $50.8 \%$ en 2008, selon l'organisation mondiale du commerce, la part de l'UE et l'ALENA dans les exportations global des accords commerciaux préférentiels est plus de $49 \%$ pour l'UE et $13 \%$ pour l'ALENA, Cette tendance faire des commerce mondiales plus concentré géographiquement.

Les accords commerciaux préférentiels (ACPr) sont, à l'OMC, des préférences commerciales unilatérales. Ils incluent le Système généralisé de préférences (au titre duquel les pays développés accordent des droits préférentiels pour des importations en provenance des pays en développement), ainsi que d'autres systèmes préférentiels non réciproques pour lesquels le Conseil général a accordé une dérogation, mais À l'OMC, les accords commerciaux régionaux (ACR) sont définis comme des accords commerciaux réciproques conclus entre deux partenaires ou plus, Ils incluent les accords de libre-échange et les unions douanières ${ }^{(10)}$.

Malgré la présence d'un grand écart entre les accords commerciaux régionaux et les principes de l'Accord général sur les tarifs douaniers et le commerce, aujourd'hui l'Organisation mondiale du commerce, Lorsque les avantages et les préférences accordées dans le cadre des accords préférentiels et régionaux sont incohérente et la clause la nation la plus favorisée (NPF).

Les négociations dans une cadre multilatérale dépendent sur les tarifs NPF, mais ces tarifs ne sont pas appliqué sur tous les pays, les pays qui sont liés avec un autre pays avec un accord ou bien arrangement régional bénéficient des droits plus inferieur aux NPF, et pour éliminer et réduire cette contradictions, en particulier dans les pays en développement été créé un système généralisé de préférences.

L'organisation mondiale du commerce a été préparé un mécanisme pour la transparence des accords commerciaux régionaux porte sur des notifications des ACPr et faire des communication des données fourni a l'OMC - son intitulé officiel, sa portée et la date de sa signature, tout calendrier prévu pour son entrée en vigueur ou son application provisoire-, selon l'article de décision pris le 14 décembre 2006, par l'OMC tout les parties de ACPr communiqueront les données sur l'accord : une liste complète des droits préférentiels, une liste complète des taux de droits NPF, marges de préférence, contingents tarifaires, restrictions saisonnières, sauvegardes spéciales et, dans la mesure du possible, équivalents ad valorem des droits non ad valorem, Règles d'origine préférentielles, Statistiques sur les importations, pour les trois années les plus récentes ${ }^{(11)}$. 
Tout les accords bilatérales de système global de préférences commerciales entre les pays en développement de l'Algérie sont notifié à l'OMC sous le type de accord de portée partielle le 25 septembre 1989, et l'accord d'association entre l'Algérie et l'UE a notifié comme accord de libre-échanges le 24 juillet 2006, tous les accords de l'Algérie sont des accords interrégional.

Les Membres de l'OMC sont toutefois autorisés à conclure des accords de ce genre sous réserve de conditions précises qui sont énoncées dans trois ensembles de règles:

- Les paragraphes 4 à 10 de l'article XXIV du GATT (tels qu'ils sont précisés dans le Mémorandum d'accord sur l'interprétation de l'article XXIV du GATT de 1994) traitent de l'établissement et du fonctionnement des unions douanières et des zones de libre-échange.

- La Clause dite d'habilitation (c'est-à-dire la Décision de 1979 intitulée Traitement différencié et plus favorable, réciprocité, et participation plus complète des pays en voie de développement) vise les arrangements commerciaux préférentiels entre pays en développement Membres.

- L'article V de l'AGCS régit la conclusion des ACR dans le domaine des services, en ce qui concerne tant les pays développés que les pays en développement.

\section{3- L'Algérie et le Nouveau système commercial}

Depuis la fin des années quatre-vingt du dernier siècle, et après la grave crise économique subie par l'Algérie, Était nécessaire de mettre des réformes urgentes, A travers de transition vers une économie de marché, Afin de dépasser les déséquilibres structurels de l'économie algérienne.

\section{3-1- L'état d'avancement de l'accession de l'Algérie a l'OMC}

L'Algérie a tenté dur pour adhérer à l'Organisation mondiale du commerce (OMC) et de la fusion dans le nouveau système commercial international, depuis 1987, date de la première demande d'adhésion à l'Accord général sur les tarifs douaniers pour tirer parti des avantages offerts par cette chaîne, en particulier pour les pays en développement, et le tableau suivant monter les différentes étapes de la processus d'accession de l'Algérie a l'OMC.

Donc, l'Algérie a fait sa demande d'adhésion au l'OMC (le GATT) le 3 juin 1987,et le groupe de travail a été établi le 17 juin 1987, l'Algérie a fourni la première Aide-mémoire le 11 juillet 1996, les questions et les réponses le 14 juillet 1996, et le groupe de travail est réuni pour la première fois en avril 1998 sous la 
direction du M.Sánchez Arnau, et a la date de 5 avril 2013 La réunion s'est tenue à sa onzième session.

\section{3-3 - L'Algérie, l'Union Européen et l'GZALE}

L'Algérie a connu des changements significatifs dans la politique commerciale a partir de 1995, où mené une politique d'économie de marché, forcé à entrer dans plusieurs arrangements commerciaux comme : l'Accord d'association avec l'Union Européenne et la Grande Zone Arabe de Libre Echange, afin de Profite des avantages et des gains de la libéralisation du commerce extérieur.

\section{3-3-1- Accord d'association Algérie avec l'Union Européenne}

L'Accord d'Association entre l'Algérie et l'Union européenne constitue le cadre des relations économiques, politiques et sociales entre les deux parties, pour les objectifs suivants : ${ }^{(12)}$

- fournir un cadre approprié au dialogue politique entre les parties afin de permettre le renforcement de leurs relations et de leur coopération dans tous les domaines qu'elles estimeront pertinents;

- développer les échanges, assurer l'essor de relations économiques et sociales équilibrées entre les parties, et fixer les conditions de la libéralisation progressive des échanges de biens, de services et de capitaux ;

- favoriser les échanges humains, notamment dans le cadre des procédures administratives ;

- encourager l'intégration maghrébine en favorisant les échanges et la coopération au sein de l'ensemble maghrébin et entre celui-ci et la Communauté européenne et ses Etats membres ;

- promouvoir la coopération dans les domaines économique, social, culturel et financier.

L'Accord d'Association signé à Valence (Espagne) en avril 2002, ne se limite pas uniquement à la création d'une zone de libre échange à l'horizon 2017 mais intègre aussi bien les aspects économiques (volet commercial, coopération économique et financière, flux d'investissements) que les dimensions politiques, sociales et culturelles nécessaires pour un développement durable, Cet accord est important car c'est avec l'UE que l'Algérie réalise près de $60 \%$ de son commerce extérieur. Il s'inscrit dans le contexte du processus de Barcelone initié par l'Union Européenne pour développer les relations de coopération avec les pays Sud Méditerranéens tout en visant la mise en place à long terme d'une zone de prospérité partagée ${ }^{(13)}$. 
Mais l'accord d'association a entré en vigueur le $1^{\text {er }}$ septembre 2005 , en vue d'établissent une zone de libre-échanges pendant une période de douze années au maximum, mais l'Algérie a demandé de reporter l'échéance de la mise en place de la Zone de libre échange à 2020 au lieu de 2017, et selon l'article 8 de l'accord d'association les produits originaires de l'Algérie sont admis à l'importation dans la Communauté en exemption de droits de douane et taxes d'effet équivalent et des restrictions quantitatives ou mesures d'effet équivalent, et les mesures appliqué par l'Algérie pour industries naissantes ou à certains secteurs ne peuvent excéder de $25 \%$.

L'Accord d'Association Algérie-UE spécifie sept volés liés aux politiques commerciales, lesquelles constituent avec les réformes économiques les priorités de la programme d'accompagnement de l'Accord d'Association dans le cadre du programme d'appui à la mise en œuvre de l'accord d'association, les politiques commerciales sont l'une des autres priorités de la programme de l'Accord d'association.

Le processus de démantèlement tarifaire pour les produits industriels a été mis en œuvre depuis le $1^{\text {er }}$ septembre 2005 à travers trois étapes de démantèlement, une première liste de 2034 lignes tarifaires a été démantelée immédiatement le 1 er septembre 2005, constituée essentiellement des matières premières et d'équipements, Une deuxième liste de 1095 lignes tarifaires, constituée de demi-produits et de certains produits finis, le démantèlement a été étalé sur 7 années avec deux années de différé pour aboutir à un taux de 0\% en 2012.

Enfin, le démantèlement de la troisième liste de 1860 lignes tarifaires constituée de produits finis a été étalé sur une période de 12 ans avec deux ans de différé pour atteindre un taux de $0 \%$ en $2017^{(14)}$, mais lors de la 5ème session du Conseil d'Association en 2010 l'Algérie a introduit une demande formelle pour la révision du démantèlement tarifaire.

\section{3-3-2- l'Algérie et la Grande Zone Arabe de Libre Echange}

La création de la grande zone arabe de libre échange Était fondée sur les recommandations du Conseil économique et social de la Ligue arabe le 19 février 1997 sous le Programme exécutif de la convention de facilitation et de développement des échanges commerciaux entre les Etat arabes faites à Tunis le 27 février 1981, où il a été décidé à établir un espace commercial entre les pays arabes dans un période de dix ans à compter du 1/1/1998, en vue de libéraliser les échanges entre les États parties et respect des règles d'origine qui sont annoncés dans Cinquante-septième session de la Conseil économique et social, échange d'informations et de données, règlement des différends et en plus traitement 
préférentiel pour les pays arabes moins avancés, la zone arabe de libre échange a été entré en vigueur le 1 Janvier 2005, mais l'Algérie a adhéré officiellement le 1 janvier 2009, après avait demandé en 2007 une période de transition. L'Algérie a élaboré une liste de 384 produits exclus de l'avantage préférentiel accordé dans le cadre de la grande zone arabe de libre échange pour des raisons religieuses, sanitaire, sécuritaires et environnementales ${ }^{(15)}$.

\section{Conclusion}

Cette contribution nous a permis de faire une analyse sur une question qui fait aujourd'hui un grand polémique, la question de incompatibilité des préférences des accords commerciaux régionaux avec les principes d'organisation mondiale du commerce.

A ce titre, il est clair que le commerce mondial sera inévitablement affecté surtout les pays en développement, bien que l'OMC a été incapable de contenir les blocs commerciaux, il n'a pas trouvé une solution sauf un mécanisme pour la transparence.

Nous avons essayé de voir la réalité de commerce extérieur et la politique commerciale de l'Algérie dans ce conflit, en 2020 l'Algérie va rentre dans la zone de liber échanges établi avec l'UE, et dans les années prochaine dans l'espace du multilatéralisme mondiale- l'OMC- et tous cela met l'Algérie face un canon d'un fusil, car l'Algérie ne bénéficiera pas des tarifs préférentiels en raison de la structure du commerce extérieur qui ne dépend que des hydrocarbures.

Pour conclure on peut dire que le monde aujourd'hui vivre Problème de confiance dans le système commercial multilatéral, et considère queles accords régionaux ces des véritables havres.

\section{Références and notes :}

Mohamed Lotfi M'rini (2005), De la Havane à Doha, Bilan juridique et commercial de l'intégration des pays en développement dans le système commercial multilatéral, les presses de l'Université de laval, canada, p 18.

Jean-Christophe GRAZ (1999), Aux sources de l'OMC : La charte de la havane 1941-1950, publications d'histoire économiques et sociale internationale, librairie Droz, Genève, Suisse, p143.

GATT (1948), Accord général sur les tarifs douaniers et le commerce, publication de GATT, secrétariat du GATT, Genève, p 55.

WTO (2011), Understanding the WTO, WTO publications, Information and external relations Division, Geneva, p16. 
Patrick LOVE, Ralph LATTIMORE (2009), Le commerce international: libre, équitable et ouvert?, les essentiels de l'OCDE, OCDE, p-p : 104-105.

OMC (2011), Rapport sur le commerce mondial 2011, l'OMC et les accords commerciaux préférentiels de la coexistence à la cohérence, publication de l'OMC, Genève, p 44.

Ibid. p-p : 52-53.

(Jean Mark Siroen, Accords commerciaux et régionalisation des échanges,la régionalisation de l'économie mondiale, http://www.dauphine.fr/siroen/acr2.pdf

OMC, Rapport sur le commerce mondial 2011, ibid. p 54.

http://www.wto.org/french/tratop_f/region_f/rta_pta_f.htm

OMC, mécanisme pour la transparence des accords commerciaux régionaux, WT/L/671, 18 décembre 2006, p-p :6-7.

Ministère du commerce, Accord euro-méditerranéen, établissant une association entre l'Algérie et l'UE, p 4.

L'accord d'association entre l'Algérie et l'UE, programme d'appui à la mise en ouvre de l'accord d'association, http://www.p3a-algerie.org/

Mustapha Benbada (2012), Allocution de Monsieur le Ministre du Commerce, Journée de sensibilisation et d'information sur le nouveau schéma de démantèlement tarifaire avec l'Union Européenne, ministère du commerce, Hôtel Sheraton, Alger, p3.

Direction de la Valeur et de la Fiscalité, circulaire $n^{\circ} 1769$ /dgd/sp/d.400 du 03 décembre 2008, direction générale des douanes, ministère des finances, p2.

\title{
Summary
}

\section{L’Algérie et les défis du commerce mondial}

\author{
Khayreddine BELAAZE \\ Mohamed Khider University, ALGERIA
}

\section{Rabah KHOUNI \\ Mohamed KhiderUniversity, ALGERIA}

This contribution aims to put light on an major challenge facing global trade today is a conflict between multilateral agreements and regional agreements, preferences granted under regional trade agreements are incompatible with the principles of World trade Organization which is the clause in the most favored nation, preferential tariffs of member states in regional agreements are often lower than MFN rates, meanwhile, the number of regional agreements has doubled since 1995 date creation of the WTO, and became a conflict between regionalism and multilateralism is imminent, Algeria through to join the World trade Organization, and the Association Agreement with the EU, GAFTA, will be in a critical position with its partners.

Keywords: Regional trade agreements, preferential arrangements, the World Trade Organization, foreign trade of Algeria. 